\title{
Analysis of PAX8 immunohistochemistry in lung cancers: a meta-analysis
}

\author{
Jae Han Jeong ${ }^{1}$, Nae Yu Kim ${ }^{2}$, Jung-Soo Pyo ${ }^{3}$ \\ 'Department of Thoracic and Cardiovascular Surgery, Chosun University Hospital, Chosun University School of Medicine, Gwangju: \\ Departments of 2Internal Medicine and ${ }^{3}$ Pathology, Daejeon Eulji University Hospital, Eulji University School of Medicine, Daejeon, Korea
}

\begin{abstract}
Background: In this meta-analysis, we aimed to evaluate the PAX8 immunohistochemical expressions in primary lung cancers and metastatic cancers to the lung. Methods: We identified and reviewed relevant articles from the PubMed databases. Ultimately, 18 articles were included in this meta-analysis. PAX8 expression rates were analyzed and compared between primary and metastatic lung cancers. Results: The PAX8 expression rate in primary lung cancers was 0.042 ( $95 \%$ confidence interval [Cl], 0.025 to 0.071$)$. PAX8 expression rates of small cell $(0.129 ; 95 \% \mathrm{Cl}, 0.022$ to 0.496$)$ and non-small cell carcinomas of the lung $(0.037 ; 95 \% \mathrm{Cl}, 0.022$ to 0.061$)$ were significantly different $(p=.049$ in a meta-regression test). However, the PAX8 expression rates of adenocarcinoma $(0.013 ; 95 \% \mathrm{Cl}, 0.006$ to $0.031)$ and squamous cell carcinoma $(0.040 ; 95 \% \mathrm{Cl}, 0.016$ to 0.097$)$ were not significantly different. PAX8 expression rates of metastatic carcinomas to the lung varied, ranging from $1.8 \%$ to $94.9 \%$. Metastatic carcinomas from the lung to other organs had a PAX8 expression rate of $6.3 \%$. The PAX8 expression rates of metastatic carcinomas from the female genital organs, kidneys, and thyroid gland to the lung were higher than those of other metastatic carcinomas. Conclusions: Primary lung cancers had a low PAX8 expression rate regardless of tumor subtype. However, the PAX8 expression rates of metastatic carcinomas from the female genital organs, kidneys, and thyroid were significantly higher than those of primary lung cancers.
\end{abstract}

Key Words: PAX8; Immunohistochemistry; Lung; Primary; Metastatic; Meta-analysis

Received: April 10, 2020 Revised: May 24, 2020 Accepted: June 8, 2020

Corresponding Author: Nae Yu Kim, MD, Department of Internal Medicine, Daejeon Eulji University Hospital, Eulji University School of Medicine, 95 Dunsanseo-ro, Seo-gu, Daejeon 35233, Korea

Tel: +82-42-611-3043, Fax: +82-42-611-3853, E-mail: naeyu46@eulji.ac.kr

Corresponding Author: Jung-Soo Pyo, MD, Department of Pathology, Daejeon Eulji University Hospital, Eulji University School of Medicine, 95 Dunsanseo-ro, Seo-gu, Daejeon 35233, Korea

Tel: +82-42-611-3895, Fax: +82-42-611-3459, E-mail: anapyojs@gmail.com

Although various targeted therapies have been developed and applied in lung cancers, a detailed diagnosis of tumor type is important to identify precise treatment [1]. Primary lung tumors includes small and non-small cell lung cancers (20\% and 80\%, respectively) [2]. However, the lung is a common metastatic site for metastatic extrapulmonary carcinomas [3]. Differential diagnosis between primary lung cancers and metastatic carcinomas to the lung is challenging in some cases due to similar histologic findings or poorly differentiated tumors [4]. Although thyroid transcription factor 1 (TTF-1) and Napsin A are useful markers for primary lung adenocarcinoma [4-7], complete differentiation between primary lung cancers and metastatic carcinomas to the lung may be limited. Additional precise markers are needed in daily practice.
The paired box transcription factor PAX8 has long been studied in malignant tumors of the lung [4,7-24]. PAX8, which includes a family of cell-lineage transcription factors, is involved in the organogenesis of the thyroid gland and kidney as well as the Müllerian system. PAX8 can be expressed in the neoplastic cells of associated organs and is expressed both in the normal tissues and tumors from these organs [25-29]. In previous studies, high PAX8 expression levels have been shown in kidney, thyroid, ovarian, endometrial, and endocervical carcinomas [25-32]. Although PAX8 expression of primary lung cancers was absent in some reports, variable PAX8 expression rates in primary lung cancers has been reported [4,7-24]. PAX8 expression in normal lung tissue is unclear. In normal lung tissue, various cell types are present, including bronchial epithelium, 
pneumocytes, submucosal glands, neuroendocrine cells, and lymphocytes, and each has a different PAX8 expression pattern. Moreover, studies have reported PAX8 expression in normal B lymphocytes $[14,18]$. These results make interpretation of PAX8 expression in the lung difficult, necessitating a meta-analysis to obtain conclusive information.

Because various malignant tumors including metastatic tumors can occur in the lung, a comparison of PAX8 expression between primary lung cancers and metastatic carcinomas to the lung is needed. In addition, detailed information based on tumor subtypes is not available. In this meta-analysis, we investigated PAX8 immunohistochemical expression rates in primary lung cancers and metastatic carcinomas to the lung as well as in various tumor subtypes of primary lung cancers. Subgroup analysis for PAX8 expression based on the origin of the metastatic carcinomas was also performed.

\section{MATERIALS AND METHODS}

\section{Database search and selection criteria}

Relevant articles were extracted from the PubMed database through January 31, 2020 using the following keywords: "PAX8," "immunohistochemistry or immunohistochemical," and "cancer or carcinoma." The titles and abstracts of all searched articles were screened. The included articles had information for PAX8 immunohistochemistry of primary lung cancers and metastatic cancers to the lung and metastatic carcinomas from the lung. Additionally, studies on metastatic lung cancers in other organs were included. However, case reports, non-original articles, or articles written in non-English were excluded. We followed PRISMA guidelines.

\section{Data extraction}

Data on the PAX8 immunohistochemical expressions of primary lung cancers and metastatic cancers to the lung and metastatic carcinomas from the lung were extracted from each eligible study [4,8-24]. All data were extracted by two independent authors. The extracted data included the authors' information, study location, number of patients analyzed, antibody information (manufacturer and clonality), tumor subtypes and PAX8 expression rates.

\section{Statistical analyses}

The meta-analysis was performed using the Comprehensive Meta-Analysis software package (Biostat, Englewood, NJ, USA). The PAX8 expression rates of various primary lung cancers and metastatic cancers to the lung and the metastatic carcinomas from the lung were investigated. In addition, subgroup analysis was performed based on tumor subtypes of the primary lung cancers and the metastatic carcinoma from the lung. Heterogeneity between studies was checked using $\mathrm{Q}$ and $\mathrm{I}^{2}$ statistics and is expressed as p-values. Additionally, sensitivity analysis was conducted to assess the heterogeneity of eligible studies and the impact of each study on the combined effects. In the meta-analysis, because the eligible studies used various evaluation criteria and PAX8 antibodies, a random-effect model was more suitable than a fixed-effect model. To assess publication bias, Begg's funnel plot and Egger's test were used. If significant publication bias was found, the fail-safe $\mathrm{N}$ and trim-fill tests were additionally used to confirm the degree of publication bias. The results were considered statistically significant at $\mathrm{p}<.05$.

\section{RESULTS}

\section{Selection and characteristics of the studies}

In this study, 457 relevant articles were extracted from the PubMed database and reviewed for meta-analysis. Of these, 265 articles with no or insufficient information for a meta-analysis and 122 non-original articles were excluded. Furthermore, 52 articles were excluded for the following reasons: in a language other than English $(n=24)$, on other diseases $(n=19)$, and involving non-human subjects $(n=9)$ (Fig. 1). Finally, 18 eligible articles were selected and included in this meta-analysis. These studies included 3,238 patients with primary and metastatic lung cancers (Table 1).

\section{PAX8 expression in primary lung cancers and metastatic cancers to the lung}

PAX8 expression rates were estimated in primary lung cancers, including small cell and non-small cell carcinomas. The PAX8 expression rate of overall primary lung cancers was $0.042(95 \%$ confidence interval [CI], 0.025 to 0.071). The PAX8 expression rates were $0.129(95 \% \mathrm{CI}, 0.022$ to 0.496$)$ and 0.037 (95\% CI, 0.022 to 0.061 ) in small cell and non-small cell carcinomas, respectively (Table 2, Fig. 2). There was a significant difference in PAX8 expression between small cell and non-small cell carcinomas by the meta-regression test $(\mathrm{p}=.049)$. Detailed subgroup analysis was performed based on tumor subtypes of non-small cell carcinomas. PAX8 expression rates were 0.013 (95\% CI, 0.006 to 0.031 ), 0.040 (95\% CI, 0.016 to 0.097 ), and 0.113 (95\% CI, 0.059 to 0.205$)$ in adenocarcinoma, squamous cell carcinoma, and large cell carcinoma, respectively. The PAX8 


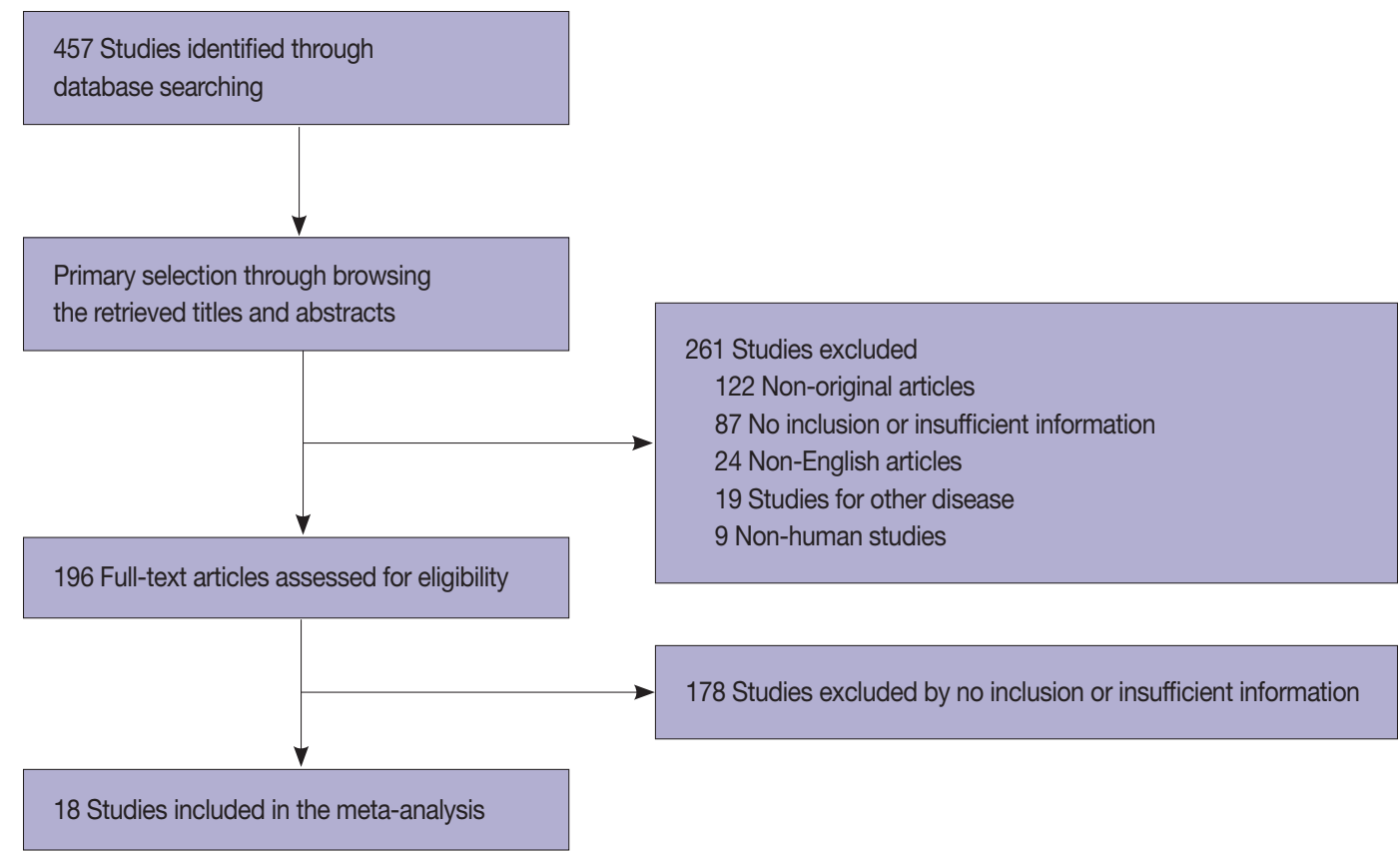

Fig. 1. Flow chart of study search and selection methods.

expression rate was 0.071 (95\% CI, 0.004 to 0.577$)$ in micropapillary carcinoma and $0.050(95 \% \mathrm{CI}, 0.007$ to 0.272$)$ in large cell neuroendocrine carcinoma. Moreover, the PAX8 expression rate of metastatic cancers from the lung to other organs was investigated; the estimated PAX8 expression rates were similar to those of primary lung cancers. The PAX8 expression rates in metastatic lung adenocarcinoma and squamous cell carcinoma were 0.050 (95\% CI, 0.003 to 0.475 ) and 0.071 (95\% CI, 0.004 to 0.577 ), respectively (Table 3 ). In addition, the PAX8 expression rate of metastatic small cell carcinomas from the lungs was 0.067 (95\% CI, 0.009 to 0.352 ).

\section{PAX8 expression in metastatic carcinomas from various organs}

The PAX8 expression rates of metastatic carcinomas to the lung were investigated to elucidate the differential role of PAX8 immunohistochemistry. Metastatic carcinomas to the lung showed different PAX8 expression rates, ranging from $1.8 \%$ to 94.9\% (Table 4, Fig. 3). Among metastatic carcinomas to the lung, PAX8 expression rates in metastatic carcinomas from the female genital organs, kidney, and thyroid were 0.746 (95\% CI, 0.501 to 0.895 ), 0.876 (95\% CI, 0.796 to 0.928 ), and 0.879 (95\% CI, 0.697 to 0.959), respectively. There were significant differences in PAX8 expression rates between primary lung cancers and metastatic carcinomas from the female genital organs, kidneys, and thyroid gland.

\section{DISCUSSION}

Because PAX8 expression can differ by tumor origin, PAX8 may be useful in differentiating malignant tumors of various origins. Some studies reported that PAX8 expression was absent or very low in primary lung cancers [4,8-24], whereas others reported high PAX8 expressions of up to $66.7 \%$ [8,14,16,21-24]; thus detailed information on PAX8 expression according to tumor subtypes of lung cancer was unclear. In this meta-analysis, we evaluated the diagnostic roles of PAX8 immunohistochemical expression in primary lung cancers and metastatic carcinomas to the lung.

It is difficult to obtain conclusive data from individual studies because of the small sample size and difference in evaluation methods. PAX8 expression was found in 0\%-66.7\%,0\%-12.5\%, and $0 \%-33.3 \%$ cases of small cell lung carcinoma, adenocarcinoma, and squamous cell carcinoma of the lung, respectively [4,8-24]. Unlike previous studies, estimated PAX8 expression rates were $12.9 \%$ and $3.6 \%$ in small cell and non-small cell carcinomas, respectively. However, the cause for discrepancy in the results between the present meta-analysis and previous studies could not be determined. Based on our results, the PAX8 expression was significantly higher in small cell carcinoma than in nonsmall cell carcinoma ( $\mathrm{p}=.049$ in the meta-regression test). Clinically, the usefulness of differentiation between small cell and non-small cell carcinomas is limited, regardless of statistical sig- 
Table 1. Main characteristics of the eligible studies

\begin{tabular}{|c|c|c|c|c|c|c|}
\hline \multirow{2}{*}{ Study } & \multirow{2}{*}{ Location } & \multirow{2}{*}{$\begin{array}{c}\text { Antibody } \\
\text { manufacturer clonality }\end{array}$} & \multirow{2}{*}{$\begin{array}{l}\text { Clonality } \\
\text { organ }\end{array}$} & \multirow{2}{*}{ Tumor subtype } & \multicolumn{2}{|c|}{ PAX8 $॥ \mathrm{HC}$} \\
\hline & & & & & Positive & Negative \\
\hline Asivatham et al. (2014) [8] & USA & Polyclonal & Lung & ND & 1 & 14 \\
\hline Bi et al. (2019) [9] & China & Monoclonal & Metastatic & & 0 & 7 \\
\hline Bi et al. (2016) [10] & China & Polyclonal & Lung & NEC & 0 & 20 \\
\hline \multirow[t]{4}{*}{ El-Maqsoud et al. (2016) [11] } & Egypt & Monoclonal & Lung & Adenocarcinoma & 0 & 30 \\
\hline & & Monoclonal & Lung & Squamous cell carcinom & 0 & 15 \\
\hline & & Monoclonal & Lung & Large cell carcinoma & 0 & 5 \\
\hline & & Monoclonal & Metastatic & & 88 & 55 \\
\hline Gailey et al. (2013) [12] & USA & Polyclonal & Lung & Squamous cell carcinoma & 0 & 12 \\
\hline Heidarpour et al. (2014) [13] & Iran & Polyclonal & Lung & $\mathrm{ND}$ & 0 & 5 \\
\hline \multirow[t]{4}{*}{ Laury et al. (2011) [14] } & USA & Polyclonal & Lung & Adenocarcinoma & 0 & 120 \\
\hline & & Polyclonal & Lung & squamous cell carcinoma & 4 & 8 \\
\hline & & Polyclonal & Lung & Adenosquamous carcinoma & 0 & 3 \\
\hline & & Polyclonal & Lung & Small cell carcinoma & 0 & 9 \\
\hline Lotan et al. (2009) [15] & USA & Polyclonal & Lung & Micropapillary carcinoma & 0 & 6 \\
\hline \multirow[t]{2}{*}{ Mentrikoski et al. (2014) [16] } & USA & Polyclonal & Lung & Adenocarcinoma & 1 & 7 \\
\hline & & Polyclonal & Lung & Squamous cell carcinoma & 0 & 3 \\
\hline \multirow[t]{3}{*}{ Nonaka et al. (2008) [17] } & USA & Polyclonal & Lung & Adenocarcinoma & 0 & 114 \\
\hline & & Polyclonal & Lung & Squamous cell carcinoma & 0 & 29 \\
\hline & & Polyclonal & Lung & Large cell carcinoma & 0 & 4 \\
\hline \multirow[t]{6}{*}{ Ozcan et al. (2011) [18] } & USA & Polyclonal & Lung & Squamous cell carcinoma & 0 & 4 \\
\hline & & Polyclonal & Lung & Adenocarcinoma & 0 & 7 \\
\hline & & Polyclonal & Lung & Small cell carcinoma & 0 & 12 \\
\hline & & Polyclonal & From lung & Small cell carcinoma & 1 & 14 \\
\hline & & Polyclonal & From lung & Adenocarcinoma & 0 & 9 \\
\hline & & Polyclonal & From lung & Squamous cell carcinoma & 0 & 6 \\
\hline Suzuki et al. (2015) [19] & Japan & Polyclonal & Lung & Squamous cell carcinoma & 0 & 5 \\
\hline Tacha et al. (2013) [20] & USA & Monoclonal & Lung & ND & 0 & 50 \\
\hline \multirow[t]{3}{*}{ Tacha et al. (2011) [21] } & USA & Polyclonal & Lung & Squamous cell carcinoma & 1 & 48 \\
\hline & & Polyclonal & Lung & Adenosquamous carcinoma & 0 & 11 \\
\hline & & Polyclonal & Lung & Adenocarcinoma & 0 & 15 \\
\hline \multirow[t]{12}{*}{ Toriyama et al. (2014) [22] } & Japan & Polyclonal & Lung & Adenocarcinoma & 6 & 247 \\
\hline & & Polyclonal & Lung & Squamous cell carcinoma & 3 & 155 \\
\hline & & Polyclonal & Lung & LCNEC & 17 & 89 \\
\hline & & Polyclonal & Lung & Small cell carcinoma & 27 & 40 \\
\hline & & Polyclonal & Lung & Pleomorphic carcinoma & 0 & 41 \\
\hline & & Polyclonal & Lung & Large cell carcinoma & 2 & 9 \\
\hline & & Monoclonal & Lung & Adenocarcinoma & 0 & 253 \\
\hline & & Monoclonal & Lung & Squamous cell carcinoma & 0 & 158 \\
\hline & & Monoclonal & Lung & LCNEC & 0 & 106 \\
\hline & & Monoclonal & Lung & Small cell carcinoma & 0 & 67 \\
\hline & & Monoclonal & Lung & Pleomorphic carcinoma & 0 & 41 \\
\hline & & Monoclonal & Lung & Large cell carcinoma & 0 & 11 \\
\hline \multirow[t]{7}{*}{ Vidarsdottir et al. (2019) [23] } & Sweden & Monoclonal & Lung & Adenocarcinoma & 2 & 429 \\
\hline & & Monoclonal & Lung & Squamous cell carcinom & 4 & 198 \\
\hline & & Monoclonal & Lung & Large cell carcinoma & 2 & 7 \\
\hline & & Monoclonal & Lung & Sarcomatoid carcinoma & 0 & 6 \\
\hline & & Monoclonal & Lung & Small cell carcinoma & 2 & 1 \\
\hline & & Monoclonal & Lung & Large cell NEC & 1 & 19 \\
\hline & & Monoclonal & Metastatic & & 55 & 251 \\
\hline Weissferdt et al. (2013) [24] & USA & Polyclonal & Lung & NEC & 2 & 23 \\
\hline \multirow[t]{2}{*}{ Ye et al. (2012) [4] } & USA & $\mathrm{ND}$ & Lung & Adenocarcinoma & 0 & 120 \\
\hline & & ND & Metastatic & & 45 & 56 \\
\hline
\end{tabular}

ND, no description; NEC, neuroendocrine carcinoma; LCNEC, large cell neuroendocrine carcinoma. 
Table 2. The estimated rates of PAX8 immunohistochemical expressions in primary lung cancers

\begin{tabular}{|c|c|c|c|c|c|}
\hline & $\begin{array}{l}\text { No. of } \\
\text { subsets }\end{array}$ & Fixed effect (95\% Cl) & $\begin{array}{l}\text { Heterogeneity test } \\
\text { p-value }\end{array}$ & Random effect $(95 \% \mathrm{Cl})$ & $\begin{array}{c}\text { Egger's test } \\
p \text {-value }\end{array}$ \\
\hline Lung, primary & 45 & $0.092(0.075-0.114)$ & $<.001$ & $0.042(0.025-0.071)$ & $<.001$ \\
\hline Small cell carcinoma & 5 & $0.350(0.254-0.461)$ & .002 & $0.129(0.022-0.496)$ & .185 \\
\hline Non-small cell carcinoma & 3,537 & $\begin{array}{l}0.054(0.042-0.071) \\
0.055(0.042-0.071)\end{array}$ & $<.001$ & $\begin{array}{l}0.036(0.021-0.061) \\
0.037(0.022-0.061)\end{array}$ & $\begin{array}{l}.022 \\
.018\end{array}$ \\
\hline Adenocarcinoma & 10 & $0.016(0.009-0.027)$ & .113 & $0.013(0.006-0.031)$ & .493 \\
\hline Micropapillary carcinoma & 1 & $0.071(0.004-0.577)$ & $>.99$ & $0.071(0.004-0.577)$ & - \\
\hline Squamous cell carcinoma & 11 & $0.041(0.025-0.067)$ & .004 & $0.040(0.016-0.097)$ & .907 \\
\hline Large cell carcinoma & 8 & 0.139 (0.094-0.199) & .216 & $0.113(0.059-0.205)$ & .101 \\
\hline Large cell NEC & 3 & $0.136(0.087-0.205)$ & .020 & $0.050(0.007-0.272)$ & .192 \\
\hline Sarcomatoid carcinoma & 1 & $0.071(0.004-0.577)$ & $>.99$ & $0.071(0.004-0.577)$ & - \\
\hline Pleomorphic carcinoma & 2 & $0.012(0.002-0.080)$ & $>.99$ & $0.012(0.002-0.080)$ & - \\
\hline Adenosquamous carcinoma & 2 & $0.071(0.010-0.373)$ & .569 & $0.071(0.010-0.373)$ & - \\
\hline
\end{tabular}

$\mathrm{Cl}$, confidence interval; $\mathrm{NEC}$, neuroendocrine carcinoma.

nificance. This may be due to the low expression rates of PAX8 in both small cell and non-small cell lung carcinomas. In addition, a significant difference in PAX8 expression between tumor subtypes of primary lung cancers was not identified in the metaregression tests. Therefore, the usefulness of PAX8 expression in the differentiation of tumor subtypes of primary lung cancer is limited.

In daily practice, pathologists have to diagnose various metastatic cancers to the lung, which is a common metastatic site for extrapulmonary carcinomas [3]. The histologic diagnosis of some metastatic cancers of unknown origin is challenging, especially when the metastatic cancer to the lung has histologic findings similar to a primary lung cancer. In previous studies, tumors that originated from the female genital organs, kidney, and thyroid gland were found to have high PAX8 expressions. PAX8 expression rates of metastatic carcinoma from these organs were $62.9 \%$ 94.9\%. However, metastatic carcinomas from other organs showed lower PAX8 expression than the metastatic carcinoma from the female genital organs, kidney, and thyroid gland (1.8\%-16.7\% vs. $62.9 \%-94.9 \%)$. Taken together, these results indicate that metastatic carcinomas from the female genital organs, kidney, and thyroid gland can be differentiated from primary lung cancers based on different PAX8 expression rates.

Because lung cancer can metastasize to other organs, PAX8 expression levels were investigated in metastatic lung cancers [33]. In this study, there was no significant difference in PAX8 expression between primary lung cancers and metastatic cancers to the lung. For example, lung adenocarcinoma metastasizes to the ovary at an extremely low rate [33]. The metastatic lung cancers can be easily differentiated from the primary ovarian cancers using immunohistochemistry for TTF-1, Napsin A, and PAX8 [7].
The most common site of distant metastasis for thyroid carcinomas is the lung [34]. In addition, some lung cancers, up to $3.1 \%$, can metastasize to the thyroid glands $[35,36]$. Thus, the need for differentiating between lung and thyroid carcinomas may be common in daily practice. The histologic findings of papillary thyroid carcinoma can overlap with some non-mucinous bronchioloalveolar and papillary types of lung adenocarcinoma [17]. Because TTF-1 is expressed in both thyroid and lung cancers, other specific markers are needed for differentiation in daily practice. In our results, PAX8 was highly expressed in metastatic thyroid cancer of the lung, similar to primary thyroid cancers [23]. According to these findings, cases with TTF-1 and PAX8 expressions theoretically can be considered as metastatic thyroid cancer. On the other hand, cases with only TTF-1 expression can be considered as primary lung cancer. The PAX8 expression rates of anaplastic thyroid carcinomas were reported to be $50.0 \%-100.0 \%$ [22,37]. In addition, the histologic findings, such as anaplastic spindle and giant cells, can overlap between pleomorphic carcinoma of the lung and anaplastic thyroid carcinoma $[1,38]$. The TTF-1 expression rates were higher in lung pleomorphic carcinoma than in anaplastic thyroid carcinoma [39,40]. Because TTF-1 expression can be lower in anaplastic thyroid carcinoma than in other types of thyroid carcinoma, the histologic findings of the original tumor are important for differentiation. Therefore, the immunohistochemical markers, including PAX8, can be useful for the differential diagnosis of malignant tumors of the lung.

PAX8 expression was evaluated by monoclonal or polyclonal antibodies. However, the difference in clonality of the primary PAX8 antibody is not clear in primary lung cancers. This point may be necessary for defining PAX8 expression because PAX8 


\begin{tabular}{|c|c|c|c|c|c|}
\hline \multirow[t]{2}{*}{$\underline{\text { Study name }}$} & \multirow[t]{2}{*}{ Subgroup within study } & \multicolumn{3}{|c|}{ Statistics for each study } & \multirow[b]{2}{*}{$\mathrm{p}$-value } \\
\hline & & $\begin{array}{c}\text { Event } \\
\text { rate }\end{array}$ & $\begin{array}{l}\text { Lower } \\
\text { limit }\end{array}$ & $\begin{array}{c}\text { Upper } \\
\text { limit }\end{array}$ & \\
\hline Laury 2011 & Small cell carcinoma & 0.050 & 0.003 & 0.475 & 0.042 \\
\hline Ozcan 2011 & Small cell carcinoma & 0.038 & 0.002 & 0.403 & 0.026 \\
\hline Toriyama 2014_M & Small cell carcinoma & 0.007 & 0.000 & 0.107 & 0.001 \\
\hline Toriyama 2014_P & Small cell carcinoma & 0.403 & 0.293 & 0.524 & 0.115 \\
\hline \multirow[t]{2}{*}{ Vidarsdottir 2019} & Small cell carcinoma & 0.667 & 0.154 & 0.957 & 0.571 \\
\hline & & 0.129 & 0.022 & 0.496 & 0.048 \\
\hline
\end{tabular}

Event rate and $95 \% \mathrm{Cl}$
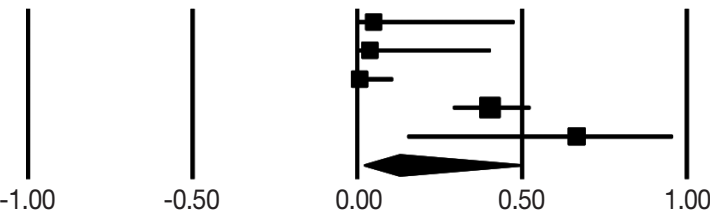

\begin{tabular}{|c|c|c|c|c|c|}
\hline \multirow[t]{2}{*}{ Study name } & \multirow[t]{2}{*}{ Subgroup within study } & \multicolumn{3}{|c|}{ Statistics for each study } & \multirow[b]{2}{*}{$\mathrm{p}$-value } \\
\hline & & $\begin{array}{c}\text { Event } \\
\text { rate }\end{array}$ & $\begin{array}{l}\text { Lower } \\
\text { limit }\end{array}$ & $\begin{array}{l}\text { Upper } \\
\text { limit }\end{array}$ & \\
\hline Вi 2016 & LCNEC & 0.024 & 0.001 & 0.287 & 0.009 \\
\hline El-Maqsoud 2016 & Adenocarcinoma & 0.016 & 0.001 & 0.211 & 0.004 \\
\hline El-Maqsoud 2016 & Large cell carcinoma & 0.083 & 0.005 & 0.622 & 0.105 \\
\hline El-Maqsoud 2016 & Squamous cell carcinoma & 0.031 & 0.002 & 0.350 & 0.017 \\
\hline Gailey 2013 & Squamous cell carcinoma & 0.038 & 0.002 & 0.403 & 0.026 \\
\hline Laury 2011 & Adenocarcinoma & 0.004 & 0.000 & 0.063 & 0.000 \\
\hline Laury 2011 & Adenosquamous carcinoma & 0.125 & 0.007 & 0.734 & 0.198 \\
\hline Laury 2011 & Squamous cell carcinoma & 0.333 & 0.131 & 0.624 & 0.258 \\
\hline Lotan 2009 & Adenocarcinoma & 0.071 & 0.004 & 0.577 & 0.081 \\
\hline Mentrikoski 2014 & Adenocarcinoma & 0.125 & 0.017 & 0.537 & 0.069 \\
\hline Mentrikoski 2014 & Squamous cell carcinoma & 0.125 & 0.007 & 0.734 & 0.198 \\
\hline Nonaka 2008 & Adenocarcinoma & 0.004 & 0.000 & 0.066 & 0.000 \\
\hline Nonaka 2008 & Large cell carcinoma & 0.100 & 0.006 & 0.674 & 0.140 \\
\hline Nonaka 2008 & Squamous cell carcinoma & 0.017 & 0.001 & 0.217 & 0.004 \\
\hline Ozcan 2011 & Adenocarcinoma & 0.063 & 0.004 & 0.539 & 0.064 \\
\hline Ozcan 2011 & Squamous cell carcinoma & 0.100 & 0.006 & 0.674 & 0.140 \\
\hline Suzuki 2015 & Squamous cell carcinoma & 0.083 & 0.005 & 0.622 & 0.105 \\
\hline Tacha 2011 & Adenocarcinoma & 0.031 & 0.002 & 0.350 & 0.017 \\
\hline Tacha 2011 & Adenosquamous carcinoma & 0.042 & 0.003 & 0.425 & 0.030 \\
\hline Tacha 2011 & Squamous cell carcinoma & 0.020 & 0.003 & 0.131 & 0.000 \\
\hline Toriyama 2014_M & Adenocarcinoma & 0.002 & 0.000 & 0.031 & 0.000 \\
\hline Toriyama 2014_M & Large cell carcinoma & 0.042 & 0.003 & 0.425 & 0.030 \\
\hline Toriyama 2014_M & LCNEC & 0.005 & 0.000 & 0.070 & 0.000 \\
\hline Toriyama 2014_M & Pleomorphic carcinoma & 0.012 & 0.001 & 0.164 & 0.002 \\
\hline Toriyama 2014_M & Squamous cell carcinoma & 0.003 & 0.000 & 0.048 & 0.000 \\
\hline Toriyama 2014_P & Adenocarcinoma & 0.024 & 0.011 & 0.052 & 0.000 \\
\hline Toriyama 2014_P & Large cell carcinoma & 0.182 & 0.046 & 0.507 & 0.054 \\
\hline Toriyama 2014_P & LCNEC & 0.160 & 0.102 & 0.243 & 0.000 \\
\hline Toriyama 2014_P & Pleomorphic carcinoma & 0.012 & 0.001 & 0.164 & 0.002 \\
\hline Toriyama 2014_P & Squamous cell carcinoma & 0.019 & 0.006 & 0.057 & 0.000 \\
\hline Vidarsdottir 2019 & Adenocarcinoma & 0.005 & 0.001 & 0.018 & 0.000 \\
\hline Vidarsdottir 2019 & Large cell carcinoma & 0.222 & 0.056 & 0.579 & 0.118 \\
\hline Vidarsdottir 2019 & LCNEC & 0.050 & 0.007 & 0.282 & 0.004 \\
\hline Vidarsdottir 2019 & Sarcomatoid carcinoma & 0.071 & 0.004 & 0.577 & 0.081 \\
\hline Vidarsdottir 2019 & Squamous cell carcinoma & 0.020 & 0.007 & 0.052 & 0.000 \\
\hline Weissferdt 2013 & NEC & 0.080 & 0.020 & 0.269 & 0.001 \\
\hline \multirow[t]{2}{*}{ Ye 2012} & Adenocarcinoma & 0.004 & 0.000 & 0.063 & 0.000 \\
\hline & & 0.037 & 0.022 & 0.061 & 0.000 \\
\hline
\end{tabular}

Fig. 2. Forest plots for PAX8 expression rates in small cell lung cancer (A) and non-small cell lung cancer (B) [4,10-12,14-19,21-24]. Cl, confidence interval; LCNEC, large cell neuroendocrine carcinoma; NEC, neuroendocrine carcinoma.

expression in primary lung cancers was low or absent. In previous studies, the immunohistochemistry using PAX8 polyclonal antibody showed cross-reactivity with other PAX families and detected immunoreactivity in normal tissues and tumors represented by B lymphocytes [41-43]. Some studies reported different PAX8 expression rates according to the clonality of anti- bodies in the same tissues [22,23]. Toriyama et al. (2014) [22] reported that PAX8 expression was only detected in immunohistochemistry with polyclonal antibody but not with a monoclonal antibody. However, Vidarsdottir et al. (2019) [23] detected PAX8 expression in primary lung cancers using a monoclonal antibody. In our results, PAX8 expression rates of small cell car- 
Table 3. The estimated rates of PAX8 immunohistochemical expressions in metastatic lung cancer

\begin{tabular}{|c|c|c|c|c|c|}
\hline & $\begin{array}{l}\text { No. of } \\
\text { subsets }\end{array}$ & Fixed effect (95\% Cl) & $\begin{array}{l}\text { Heterogeneity test } \\
\text { p-value }\end{array}$ & Random effect $(95 \% \mathrm{Cl})$ & $\begin{array}{c}\text { Egger's test } \\
p \text {-value }\end{array}$ \\
\hline Lung, metastatic & 3 & $0.063(0.016-0.220)$ & .980 & $0.063(0.016-0.220)$ & .755 \\
\hline Adenocarcinoma & 1 & $0.050(0.003-0.475)$ & $>.99$ & $0.050(0.003-0.475)$ & - \\
\hline Squamous cell carcinoma & 1 & $0.071(0.004-0.577)$ & $>.99$ & $0.071(0.004-0.577)$ & - \\
\hline Small cell carcinoma & 1 & 0.067 (0.009-0.352) & $>.99$ & $0.067(0.009-0.352)$ & - \\
\hline
\end{tabular}

$\mathrm{Cl}$, confidence interval.

Table 4. The estimated rates of PAX8 immunohistochemical expressions in metastatic carcinoma to the lung

\begin{tabular}{|c|c|c|c|c|c|}
\hline & $\begin{array}{l}\text { No. of } \\
\text { subsets }\end{array}$ & Fixed effect (95\% Cl) & $\begin{array}{l}\text { Heterogeneity test } \\
\text { p-value }\end{array}$ & Random effect $(95 \% \mathrm{Cl})$ & $\begin{array}{c}\text { Egger's test } \\
p \text {-value }\end{array}$ \\
\hline Metastatic carcinoma & 39 & $0.492(0.412-0.573)$ & $<.001$ & $0.337(0.191-0.523)$ & .005 \\
\hline Adrenal cortex & 1 & $0.167(0.010-0.806)$ & $>.99$ & $0.167(0.010-0.806)$ & - \\
\hline Appendix & 1 & $0.100(0.006-0.674)$ & $>.99$ & $0.100(0.006-0.674)$ & - \\
\hline Breast & 3 & $0.039(0.010-0.142)$ & .991 & $0.039(0.010-0.142)$ & .701 \\
\hline Colorectum & 3 & $0.018(0.006-0.056)$ & .468 & $0.018(0.006-0.056)$ & .049 \\
\hline Esophagus & 1 & $0.125(0.007-0.734)$ & $>.99$ & $0.125(0.007-0.734)$ & - \\
\hline Female genital organ ${ }^{a}$ & 7 & 0.717 (0.571-0.828) & .042 & $0.746(0.501-0.895)$ & .570 \\
\hline Ovary & 4 & $0.641(0.423-0.813)$ & .038 & 0.629 (0.243-0.899) & .908 \\
\hline Mucinous carcinoma & 1 & $0.600(0.297-0.842)$ & $>.99$ & $0.600(0.297-0.842)$ & - \\
\hline Serous carcinoma & 1 & $0.955(0.552-0.997)$ & $>.99$ & $0.955(0.552-0.997)$ & - \\
\hline Uterine endometrium & 2 & 0.949 (0.713-0.993) & .615 & 0.949 (0.713-0.993) & - \\
\hline Kidney-RCC & 5 & $0.876(0.796-0.928)$ & .884 & $0.876(0.796-0.928)$ & .213 \\
\hline Clear cell RCC & 1 & 0.923 (0.609-0.989) & $>.99$ & 0.923 (0.609-0.989) & - \\
\hline Chromophobe RCC & 1 & $0.833(0.369-0.977)$ & $>.99$ & $0.833(0.369-0.977)$ & - \\
\hline Papillary RCC & 1 & $0.958(0.575-0.997)$ & $>.99$ & $0.958(0.575-0.997)$ & - \\
\hline Liver & 3 & $0.063(0.013-0.260)$ & .914 & $0.063(0.013-0.260)$ & .048 \\
\hline Pancreatobiliary & 2 & $0.133(0.026-0.466)$ & .472 & $0.133(0.026-0.466)$ & - \\
\hline Prostate & 2 & $0.072(0.014-0.290)$ & .678 & $0.072(0.014-0.290)$ & - \\
\hline Salivary gland & 2 & $0.071(0.010-0.370)$ & $>.99$ & $0.071(0.010-0.370)$ & - \\
\hline Small bowel & 1 & $0.167(0.010-0.806)$ & $>.99$ & $0.167(0.010-0.806)$ & - \\
\hline Stomach & 1 & $0.083(0.005-0.622)$ & $>.99$ & $0.083(0.005-0.622)$ & - \\
\hline Testis & 1 & $0.125(0.007-0.734)$ & $>.99$ & $0.125(0.007-0.734)$ & - \\
\hline Thyroid & 4 & $0.879(0.697-0.959)$ & .421 & 0.879 (0.697-0.959) & .839 \\
\hline Papillary carcinoma & 1 & $0.938(0.665-0.991)$ & $>.99$ & $0.938(0.665-0.991)$ & - \\
\hline Follicular carcinoma & 1 & $0.967(0.634-0.998)$ & $>.99$ & $0.967(0.634-0.998)$ & - \\
\hline Urinary system & 2 & $0.171(0.032-0.564)$ & .261 & $0.165(0.024-0.613)$ & - \\
\hline
\end{tabular}

$\mathrm{Cl}$, confidence interval; $\mathrm{RCC}$, renal cell carcinoma.

alncluded uterine corpus and cervix and ovary.

cinoma were 0.113 (95\% CI, 0.001 to 0.968 ) and 0.141 (95\% CI, 0.019 to 0.585$)$ in monoclonal and polyclonal antibodies, respectively (data not shown). In addition, in non-small cell lung carcinoma, PAX8 expression rates were 0.020 (95\% CI, 0.009 to 0.044 ) and 0.057 (95\% CI, 0.033 to 0.095 ) in monoclonal and polyclonal antibodies, respectively (data not shown). However, the difference in PAX8 expression between clonalities of PAX8 antibody was significant in non-small cell lung carcinoma but not in small cell lung carcinoma $(\mathrm{p}=.030$ and $\mathrm{p}=$ .993 in the meta-regression test, respectively). Therefore, the difference in PAX8 expression rates as detected by monoclonal and polyclonal antibodies is uncertain. However, regardless of these differences, both monoclonal and polyclonal antibodies can be useful for differentiating between metastatic carcinomas with high expression rates of PAX8 and primary lung cancers with low expression rates of PAX8. From previous studies, the high expression rate of PAX8 immunohistochemistry using a polyclonal antibody can be considered a highly sensitive method. Conversely, the low expression rate of PAX8 immunohistochemistry using a monoclonal antibody can be considered a highly specific method. As described above, the possibility of misinterpretation for other components, including normally 


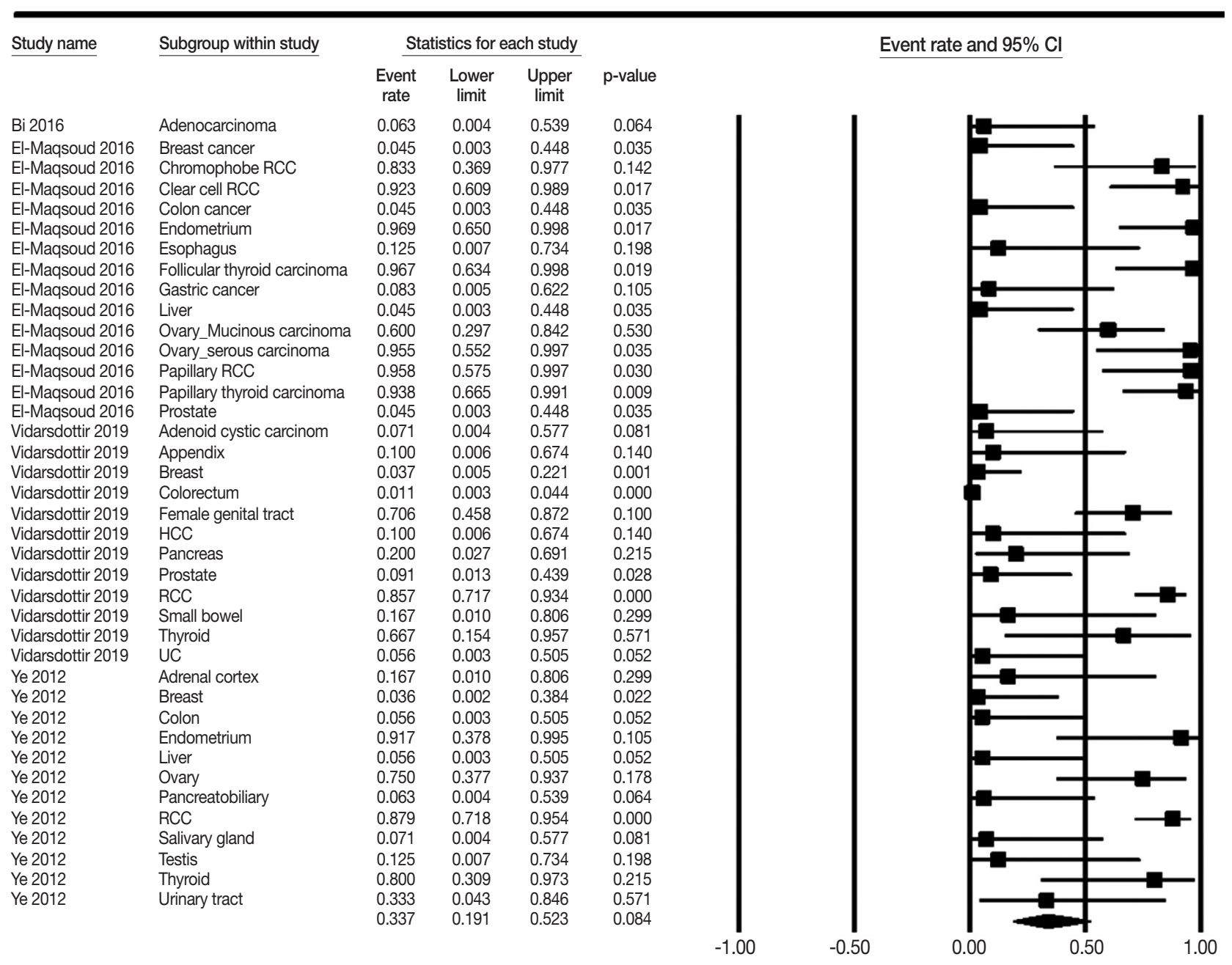

Fig. 3. Forest plot for $P A X 8$ expression rate in metastatic carcinomas to the lung from other organs $[4,10,11,23]$. $\mathrm{Cl}$, confidence interval; RCC, renal cell carcinoma; HCC, hepatocellular carcinoma.

placed components, should be considered. In addition, immunohistochemical staining techniques can affect the positive rates of PAX8, regardless of the clonality of antibody.

This study has some limitations. First, subgroup analysis based on clonality of antibody could not be performed for metastatic carcinomas from other organs due to insufficient information. Second, the detailed information for PAX8 expression of metastatic lung cancer based on metastatic organs could not be obtained due to insufficient information. Third, although the pleura is the most common metastatic site of lung cancer, a comparison of PAX8 expressions between the primary and metastatic tumors of the pleura could not be performed due to a lack of information. Fourth, subgroup analysis based on differentiation of tumors and subtypes of adenocarcinoma could not be performed due to insufficient information on eligible studies. Fifth, detailed analyses for some uncommon subtypes of the pri- mary lung cancer with low incidence were not performed due to the small sample size of patients with these cancers. Lastly, the impact of changed diagnostic criteria of primary lung cancers could not be investigated because we did not include cases applying revised diagnostic criteria in eligible studies.

In conclusion, our results showed that PAX8 expression was lower in primary lung cancer than in metastatic carcinoma of the lung. However, the diagnostic role of PAX8 expression in differentiating tumor subtypes of primary lung cancers is limited due to low rates of PAX8 immunohistochemistry. In daily practice, PAX8 immunohistochemistry can be useful for the diagnosis of metastatic cancer from the female genital organs, kidney, and thyroid gland.

\section{Ethics Statement}

Not applicable. 


\section{ORCID}

Jae Han Jeong https://orcid.org/0000-0003-1112-7405

Nae Yu Kim https://orcid.org/0000-0002-0461-6385

Jung-Soo Pyo https://orcid.org/0000-0003-0320-8088

\section{Author Contributions}

Conceptualization: JHJ, JSP. Data curation: JSP. Formal analysis: JHJ, NYK. Funding acquisition: JHJ. Investigation: JHJ, JSP. Methodology: JSP. Supervision: NYK. Validation: NYK. Writing—original draft: JHJ, JSP. Writing— review \& editing: NYK. Approval of final manuscript: all authors.

\section{Conflicts of Interest}

The authors declare that they have no potential conflicts of interest.

\section{Funding Statement}

This study was supported by research fund from Chosun University Hospital, 2019.

\section{References}

1. Travis WD, Brambilla E, Burke AP, Marx A, Nicholson AG. WHO classification of tumours of the lung, pleura, thymus and heart. 4th ed. Lyon: International Agency for Research on Cancer, 2015.

2. Brown AF, Sirohi D, Fukuoka J, et al. Tissue-preserving antibody cocktails to differentiate primary squamous cell carcinoma, adenocarcinoma, and small cell carcinoma of lung. Arch Pathol Lab Med 2013; 137: 1274-81.

3. Travis WD. Pathology of lung cancer. Clin Chest Med 2002; 23: 6581.

4. Ye J, Hameed O, Findeis-Hosey JJ, et al. Diagnostic utility of PAX8, TTF-1 and napsin A for discriminating metastatic carcinoma from primary adenocarcinoma of the lung. Biotech Histochem 2012; 87: 30-4.

5. Bishop JA, Sharma R, Illei PB. Napsin A and thyroid transcription factor-1 expression in carcinomas of the lung, breast, pancreas, colon, kidney, thyroid, and malignant mesothelioma. Hum Pathol 2010; 41: 20-5.

6. Boggaram V. Thyroid transcription factor-1 (TTF-1/Nkx2.1/TITF1) gene regulation in the lung. Clin Sci (Lond) 2009; 116: 27-35.

7. Ye J, Findeis-Hosey JJ, Yang Q, et al. Combination of napsin A and TTF-1 immunohistochemistry helps in differentiating primary lung adenocarcinoma from metastatic carcinoma in the lung. Appl Immunohistochem Mol Morphol 2011; 19: 313-7.

8. Asirvatham JR, Esposito MJ, Bhuiya TA. Role of PAX-8, CD5, and $\mathrm{CD} 117$ in distinguishing thymic carcinoma from poorly differentiated lung carcinoma. Appl Immunohistochem Mol Morphol 2014; 22: $372-6$

9. Bi R, Bai Q, Zhu X, et al. ALK rearrangement: a high-frequency alteration in ovarian metastasis from lung adenocarcinoma. Diagn Pathol 2019; 14: 96.

10. Bi Y, Deng Y, Li S, et al. Immunophenotypic and prognostic analysis of PAX8 and TTF-1 expressions in neuroendocrine carcinomas of thymic origin: A comparative study with their pulmonary counterparts. J Surg Oncol 2016; 114: 697-702.

11. El-Maqsoud NM, Tawfiek ER, Abdelmeged A, Rahman MF, Moustafa AA. The diagnostic utility of the triple markers Napsin A, TTF-1, and PAX8 in differentiating between primary and metastatic lung carcinomas. Tumour Biol 2016; 37: 3123-34.

12. Gailey MP, Bellizzi AM. Immunohistochemistry for the novel markers glypican 3, PAX8, and p40 (DeltaNp63) in squamous cell and urothelial carcinoma. Am J Clin Pathol 2013; 140: 872-80.

13. Heidarpour M, Tavanafar Z. Diagnostic utility of PAX8 in differentiation of mullerian from non-mullerian tumors. Adv Biomed Res 2014; 3: 96.

14. Laury AR, Perets R, Piao H, et al. A comprehensive analysis of PAX8 expression in human epithelial tumors. Am J Surg Pathol 2011; 35: 816-26.

15. Lotan TL, Ye H, Melamed J, Wu XR, Shih Ie M, Epstein JI. Immunohistochemical panel to identify the primary site of invasive micropapillary carcinoma. Am J Surg Pathol 2009; 33: 1037-41.

16. Mentrikoski MJ, Wendroth SM, Wick MR. Immunohistochemical distinction of renal cell carcinoma from other carcinomas with clear-cell histomorphology: utility of CD10 and CA-125 in addition to PAX-2, PAX-8, RCCma, and adipophilin. Appl Immunohistochem Mol Morphol 2014; 22: 635-41.

17. Nonaka D, Tang Y, Chiriboga L, Rivera M, Ghossein R. Diagnostic utility of thyroid transcription factors Pax8 and TTF-2 (FoxE1) in thyroid epithelial neoplasms. Mod Pathol 2008; 21: 192-200.

18. Ozcan A, Shen SS, Hamilton C, et al. PAX 8 expression in nonneoplastic tissues, primary tumors, and metastatic tumors: a comprehensive immunohistochemical study. Mod Pathol 2011; 24: 751-64.

19. Suzuki A, Hirokawa M, Takada N, et al. Diagnostic significance of PAX8 in thyroid squamous cell carcinoma. Endocr J 2015; 62: 991-5.

20. Tacha D, Qi W, Zhou D, Bremer R, Cheng L. PAX8 mouse monoclonal antibody [BC12] recognizes a restricted epitope and is highly sensitive in renal cell and ovarian cancers but does not cross-react with $\mathrm{b}$ cells and tumors of pancreatic origin. Appl Immunohistochem Mol Morphol 2013; 21: 59-63.

21. Tacha D, Zhou D, Cheng L. Expression of PAX8 in normal and neoplastic tissues: a comprehensive immunohistochemical study. Appl Immunohistochem Mol Morphol 2011; 19: 293-9.

22. Toriyama A, Mori T, Sekine S, Yoshida A, Hino O, Tsuta K. Utility of PAX8 mouse monoclonal antibody in the diagnosis of thyroid, thymic, pleural and lung tumours: a comparison with polyclonal PAX8 antibody. Histopathology 2014; 65: 465-72.

23. Vidarsdottir H, Tran L, Nodin B, et al. Immunohistochemical profiles in primary lung cancers and epithelial pulmonary metastases. Hum Pathol 2019; 84: 221-30.

24. Weissferdt A, Tang X, Wistuba, II, Moran CA. Comparative immunohistochemical analysis of pulmonary and thymic neuroendocrine carcinomas using PAX8 and TTF-1. Mod Pathol 2013; 26: 1554-60.

25. Bowen NJ, Logani S, Dickerson EB, et al. Emerging roles for PAX8 in ovarian cancer and endosalpingeal development. Gynecol Oncol 2007; 104: 331-7.

26. Kobel M, Kalloger SE, Boyd N, et al. Ovarian carcinoma subtypes are different diseases: implications for biomarker studies. PLoS Med 2008; 5: e232.

27. Nonaka D, Chiriboga L, Soslow RA. Expression of pax8 as a useful marker in distinguishing ovarian carcinomas from mammary carcinomas. Am J Surg Pathol 2008; 32: 1566-71.

28. Tong GX, Weeden EM, Hamele-Bena D, et al. Expression of PAX8 in nephrogenic adenoma and clear cell adenocarcinoma of the lower urinary tract: evidence of related histogenesis? Am J Surg Pathol 2008; 32: 1380-7.

29. Tong GX, Yu WM, Beaubier NT, et al. Expression of PAX8 in nor- 
mal and neoplastic renal tissues: an immunohistochemical study. Mod Pathol 2009; 22: 1218-27.

30. Albadine R, Schultz L, Illei P, et al. PAX8 (+)/p63 (-) immunostaining pattern in renal collecting duct carcinoma (CDC): a useful immunoprofile in the differential diagnosis of CDC versus urothelial carcinoma of upper urinary tract. Am J Surg Pathol 2010; 34: 965-9.

31. Fabbro D, Di Loreto C, Beltrami CA, Belfiore A, Di Lauro R, Damante G. Expression of thyroid-specific transcription factors TTF-1 and PAX-8 in human thyroid neoplasms. Cancer Res 1994; 54: 4744-9.

32. Fujiwara M, Taube J, Sharma M, McCalmont TH, Kim J. PAX8 discriminates ovarian metastases from adnexal tumors and other cutaneous metastases. J Cutan Pathol 2010; 37: 938-43.

33. Niu FY, Zhou Q, Yang JJ, et al. Distribution and prognosis of uncommon metastases from non-small cell lung cancer. BMC Cancer 2016; 16: 149.

34. Mazzaferri EL. Thyroid carcinoma: papillary and follicular. In: Mazzaferri EL, Samaan N, eds. Endocrine tumors. Cambridge: Blackwell Scientific Publications Inc., 1993; 278-333.

35. Lin JD, Weng HF, Ho YS. Clinical and pathological characteristics of secondary thyroid cancer. Thyroid 1998; 8: 149-53.

36. Nakhjavani MK, Gharib H, Goellner JR, van Heerden JA. Metastasis to the thyroid gland: a report of 43 cases. Cancer 1997; 79: 574-8.

37. Kuhn E, Ragazzi M, Ciarrocchi A, et al. Angiosarcoma and anaplastic carcinoma of the thyroid are two distinct entities: a morphologic, immunohistochemical, and genetic study. Mod Pathol 2019;
32: 787-98.

38. Colby T, Koss M, Travis W. Carcinoma of the lung; clinical, radiographic aspects, spread, staging, management, and prognosis. In: Colby T, Koss M, Travis W, eds. Tumors of the lower respiratory tract, 3rd series edition. Washington, DC: Armed Forces Institute of Pathology, 1995; 107-34.

39. Miettinen M, Franssila KO. Variable expression of keratins and nearly uniform lack of thyroid transcription factor 1 in thyroid anaplastic carcinoma. Hum Pathol 2000; 31: 1139-45.

40. Rossi G, Cavazza A, Sturm N, et al. Pulmonary carcinomas with pleomorphic, sarcomatoid, or sarcomatous elements: a clinicopathologic and immunohistochemical study of 75 cases. Am J Surg Pathol 2003; 27: 311-24.

41. Moretti L, Medeiros LJ, Kunkalla K, Williams MD, Singh RR, Vega F. N-terminal PAX8 polyclonal antibody shows cross-reactivity with N-terminal region of PAX5 and is responsible for reports of PAX8 positivity in malignant lymphomas. Mod Pathol 2012; 25: 231-6.

42. Morgan EA, Pozdnyakova O, Nascimento AF, Hirsch MS. PAX8 and PAX5 are differentially expressed in B-cell and T-cell lymphomas. Histopathology 2013; 62: 406-13.

43. Lorenzo PI, Jimenez Moreno CM, Delgado I, et al. Immunohistochemical assessment of Pax8 expression during pancreatic islet development and in human neuroendocrine tumors. Histochem Cell Biol 2011; 136: 595-607. 